\title{
Original article (Full paper) \\ The effect of different training modalities on physical fitness in women over 50 years of age
}

\author{
Lucas Melo Neves \\ Tiego Aparecido Diniz \\ Universidade de São Paulo, São Paulo, SP, Brasil \\ Fabrício Eduardo Rossi \\ Ana Claudia de Souza Fortaleza \\ Universidade Estadual Paulista "Júlio de Mesquita Filho", Rio Claro, SP, Brasil \\ Eduardo Tomoyoshi Horimoto \\ Vanessa de Oliveira Geraldo \\ Universidade Estadual Paulista “Júlio de Mesquita Filho", Presidente Prudente, SP, Brasil \\ Vanessa Ribeiro dos Santos \\ Universidade Estadual Paulista "Júlio de Mesquita Filho”, Rio Claro, SP, Brasil \\ Ismael Forte Freitas Júnior \\ Universidade Estadual Paulista "Júlio de Mesquita Filho", Rio Claro, SP, Brasil \\ Universidade Estadual Paulista "Júlio de Mesquita Filho", Presidente Prudente, SP, Brasil
}

\begin{abstract}
It is not yet clear which physical exercise protocols have greater potential to mitigate the functional decline in physical fitness (coordination, flexibility, strength, agility and cardiorespiratory capacity). Thus, the objective of this study was to compare different training modalities on physical fitness in women over 50 years of age and understand which training protocol is more effective for promoting significant improvements in physical fitness. The initial sample consisted of 79 subjects, of which 17 dropped out, resulting in a total of 62 subjects who completed the study and are included in the analysis $(n=62)$. The average age of the study population was $60.1 \pm 5.4$ years, divided into five groups: aerobic training, concurrent training, multimodal training, functional training and control group. Physical fitness was analyzed through AAHPERD (American Alliance for Health, Physical Education, Recreation and Dance) tests (0 and eighth weeks). The ANOVA analyses (aleatory effect) show a main effect of time for coordination $(p<0.001)$, strength $(p=0.004)$, agility $(p<0.001)$ and cardiorespiratory capacity $(p<0.001)$, and statistically significant interactions for coordination $(p=0.003)$ and cardiorespiratory capacity $(p=0.033)$. When comparing the average relative differences between the group $(\Delta \%)$, significant improvement was observed in coordination and agility $(p<0.001)$ in the multimodal and functional training groups. For cardiorespiratory fitness, there was a significant improvement in the aerobic, concurrent and multimodal training groups $(p<0.001)$ and strength increased in the functional training group $(p=0.04)$. Functional training was demonstrated to be more effective at promoting significant improvements in physical fitness than the other investigated exercise protocols.
\end{abstract}

Keywords: AAHPERD; activities of daily living; exercise

\section{Introduction}

Aging associated with physical inactivity can result in decreases in strength and aerobic fitness ${ }^{1,2}$, and can be associated with physical fitness ${ }^{3}$, defined as the individual's physical capacity to perform ordinary and unexpected demands of daily life safely and effectively ${ }^{4}$, or the ability to carry out daily tasks with vigor and alertness, without undue fatigue and with ample energy to enjoy leisure-time pursuits and meet unforeseen emergencies ${ }^{5}$. Strength, cardiorespiratory capacity, balance, gait speed and manual dexterity levels represent a positive relationship with best performance daily activities and greater individual autonomy?
The practice of physical exercise is one of the strategies to attenuate the commonly observed decline in functional fitness ${ }^{7-11}$. Several studies have shown that physical exercise contributes to improvement in functional fitness ${ }^{7,8,11}$. Purath and collaborators found that physical exercise prescription based on functional fitness level (strength, endurance and balance) was effective at improving these variables ${ }^{12}$. Despite this statement seeming to be redundant, this study demonstrates the potential of self-directed exercises, variables that can influence functional ability in the elderly. In contrast to this statement, Cadore and collaborators demonstrated that training involving a combination of strength and endurance in elderly individuals (concurrent training) is the most suitable strategy for improving both neuromuscular 
and cardiopulmonary functions, and consequently maintaining functional capacity during aging ${ }^{13}$.

Several other studies have investigated how exercise can mitigate the decline in functional fitness, exploring modalities such as vibrating platforms ${ }^{7}$ and strength training ${ }^{10}$. Therefore, it is unclear which physical exercise strategies enhance the improvement in functional fitness. There is now a consensus regarding a need for research to compare the effects of different types of intervention on physical fitness ${ }^{7-12}$.

Thus, the objective of this study was to compare the effects of different training modalities on coordination, flexibility, strength, agility, and cardiorespiratory capacity in women over 50 years of age and understand which type of training is more effective for promoting significant improvements in physical fitness.

\section{Methods}

\section{Design}

This study is a longitudinal prospective research study (four intervention groups and one control group), and was developed in the Motor Activity Research Lab and Prescription CenterCELAPAM - in the Department of Physical Education, College of Science and Technology, FCT UNESP, Presidente Prudente campus, Brazil.

\section{Subjects}

The study population consisted of women between 50 and 70 years of age, living in Presidente Prudente, São Paulo, Brazil. The participants were invited via newspapers, radio and television adverts. The collection period was between January 2013 and December 2014. As this was the first intervention study in our laboratory with physical fitness as the primary outcome measured using the AAHPERD (American Alliance for Health, Physical Education, Recreation and Dance) battery tests, it was established that the groups required a minimum of 12 subjects to start the intervention. A total of 149 subjects were evaluated during the period of the survey, of which 79 were selected and targeted for intervention. The inclusion of the participants took place every 6 months. Aerobic group (initial moment $=12$; dropout $=3$; analyzed subjects $=9$; January 2013), functional group $($ initial moment $=20$; dropout $=6$; analyzed subjects $=14$; July 2013), control group (initial moment $=20$; dropout $=4$; analyzed subjects $=16$; July 2013), multimodal group (initial moment $=15$; dropout $=1$; analyzed subjects $=14 ;$ Jan 2014) and concurrent group (initial moment $=12$; dropout $=3$; analyzed subjects $=9$; July 2014). After the 8-week intervention or monitoring (control group), the assessments were performed again.

In order to take part in the study, the participants were required not to have performed any kind of physical exercise in the 6 months prior to the initiation of this study, in addition to not having any motor problems, musculoskeletal injuries or other comorbidities that would inhibit performing the exercises in the protocol, confirmed by a physician.
This study was approved by the Research Ethics Committee of Universidade Estadual Paulista - UNESP (FCT-UNESP Presidente Prudente, Brazil) through the Ethics Assessment Certificate (CAAE) Protocol No. 11547013.2.0000.5402. All participants who agreed to take part in the study signed the Informed Consent form and the research was conducted in accordance with the Helsinki Declaration, revised 2008.

\section{Measurements}

\section{Anthropometry and Body Composition}

For the anthropometric measures, all participants wore light clothing and were barefoot. Height was measured using a fixed stadiometer (Sanny, São Bernardo do Campo, São Paulo, Brazil), with an accuracy of $0.1 \mathrm{~cm}$. The body weight measurement was performed using a digital scale (Filizola PL 50, Filizzola Ltda., Brazil), with an accuracy of $0.1 \mathrm{~kg}$. Body mass index (BMI) was calculated using weight $/$ height $^{2}\left(\mathrm{~kg} / \mathrm{cm}^{2}\right)$.

Body composition was estimated using a DXA scanner, version 4.7 (Lunar DPX-NT; General Electric Healthcare, Little Chalfont, Buckinghamshire, United Kingdom). The subjects remained immobile in a supine position throughout the examination. Total body fat mass was expressed in absolute $(\mathrm{kg})$ and relative $(\%)$ values. All measurements were carried out at the university laboratory in a temperature-controlled room. Each morning, before the beginning of the measurements, the equipment was calibrated by the same researcher, according to the manufacturer's instructions. These results were calculated using specific software supplied with the equipment (EnCORE version 11.x, Madsion, Wincosin, United States).

\section{Functional Fitness Assessment}

The set of tests used to assess functional ability was proposed by the AAHPERD and consists of five motor tests. The parameters evaluated in this set of tests are closely related to the functional independence of the individual, with better performance representing greater autonomy. The indirectly evaluated variables are as follows: agility and dynamic balance, coordination, flexibility, strength endurance and aerobic endurance ${ }^{4,14}$.

\section{Tests Description:}

1 Agility and dynamic balance test: The participant begins the test sitting on a chair with their heels on the ground. At a signal from the evaluator, the individual rises from the chair and moves to the right, around a cone, before returning and sitting down again, repeating the movement to the left side. The distance between the cone and the chair is 1.8 meters. The test consists of moving around the cones to the right and left twice, with a total of four actions. Two attempts were allowed at the test and the best performance considered in the analysis. 
2 Coordination test: The participants sit in front of a table and use their dominant hand to perform the test. Three cans are placed on the table, (on the right for the right-handed individuals and on the left for the left-handers), the participant is required to move the cans to the next position, one by one, considering the reversal of the three cans as one turn. The test consists of four turns (12 cans moved) as a complete circuit. The shortest circuit time is considered for the final result.

3 Flexibility test: The participant sits on the floor with their legs extended, positioned on a tape affixed to the ground. The individual then reaches forward as far as possible and the displacement distance is measured while the participant remains in the final position. The subjects have two practical attempts. The final result is the best performance from two attempts.

4 Strength endurance test: With the dominant arm, the participant contracts the biceps by performing an elbow flexion, performing as many repetitions as possible in 30 seconds. The final result is the best performance from two attempts.

5 General aerobic capacity test: The participant is instructed to walk $804.67 \mathrm{~m}$ as quickly as possible, without running, on a 400-meter running track. Time is recorded in minutes and seconds and converted to seconds.

\section{Training protocol}

The training protocol consisted of an 8-week intervention with three sessions per week on nonconsecutive days. Prior to beginning the intervention, a 2 -week familiarization period was allowed, using the same equipment and procedures as the training sessions. The sessions were 50 and 70 minutes in length (Chart 1). Only participants with a minimum of $85 \%$ attendance in the training sessions were included in the analyses. Different training times between the protocols (the time between 50 and 70 minutes) were used as it has been demonstrated in previous studies by our group that equalization of load between aerobic training (walking) and concurrent training requires different stimulus times ${ }^{15}$. Previous equalization between all protocols was not possible in this study, and we used the references from the available data to guide our proposal.

\section{Intervention 1: Aerobic training}

The aerobic training protocol consisted of walking on an athletic track for 50 minutes. Determination of the aerobic training intensity was performed using the Critical Velocity (CV) protocol proposed by Wakayoshi ${ }^{16,17}$. This method was used to determine the aerobic activity for the concurrent, multimodal and functional training intensity. The groups traveled three distances $(400,800$ and $1200 \mathrm{~m})$ on a running track on nonconsecutive days. The participants were instructed to cover the distance in the shortest possible time, which was recorded using a digital stopwatch (Polar $\left.{ }^{\circledR}\right)$. A linear regression was obtained by the relation between distance (meters) and time (seconds). The $\mathrm{CV}$ was assumed as the angular coefficient of the linear regression straight line between the distances and the respective times obtained in each repetition ${ }^{16,17}$. Prior to beginning the training the participants performed 2 weeks of familiarization, which consisted of walking gradually until they reached the training volume (50 minutes). The training volume was equivalent to 50 minutes/day at $100 \% \mathrm{CV}$. This time was chosen as the total time during the week because recommendation for a person to be considered active by the American College of Sports Medicine (ACSM) is 150 minutes per week (or 50 minutes three times per week $)^{18}$. The method of intensity determination of aerobic training was also applied in the walking session of the other training protocols, (functional, multimodal and concurrent training). Each exercise session included a 5-10 minute warm-up and a 5-10 minute cool down. After 4 weeks, the $\mathrm{CV}$ procedures were

Chart 1: Summary of the interventions

\begin{tabular}{|c|c|c|c|c|c|}
\hline & Exercise & Session Details & Session time & Rest interval & Training load \\
\hline $\begin{array}{l}\text { Intervention 1: } \\
\text { Aerobic training }\end{array}$ & Aerobic & $\begin{array}{l}\text { Walking on athletic track } \\
(400 \mathrm{~m})\end{array}$ & 50 ' & None & $100 \%$ Critical velocity \\
\hline \multirow{2}{*}{$\begin{array}{l}\text { Intervention } \\
\text { 2: Functional } \\
\text { training }\end{array}$} & Functional & $\begin{array}{l}11 \text { exercises for strength } \\
\text { with training equipment }\end{array}$ & $40^{\prime}$ & $30^{\prime}$ & $\begin{array}{l}\text { Weeks 1-4: three sets circuit-stimulus } 40 \text { " each } \\
\text { base (BORG } 12 \text { and 13) } \\
\text { Weeks 5-8: three circuit sets-stimulus 50" each } \\
\text { base (BORG } 12 \text { and 13) }\end{array}$ \\
\hline & Aerobic & $\begin{array}{l}\text { Walking on athletic track } \\
(400 \mathrm{~m})\end{array}$ & $15^{\prime}$ & None & $100 \%$ Critical velocity \\
\hline \multirow{2}{*}{$\begin{array}{l}\text { Intervention } \\
\text { 3: Concurrent } \\
\text { training }\end{array}$} & Strength & $\begin{array}{l}11 \text { exercises for strength } \\
\text { with training equipment }\end{array}$ & $27^{\prime}$ & 1 ' & $\begin{array}{l}\text { Weeks } 1 \text { to } 4: 3 \times 12-15 \text { repetitions } 65-70 \% 1 \mathrm{RM} \\
\text { Weeks } 5-8: 3 \times 10-12 \text { repetitions } 70-75 \% 1 \mathrm{RM}\end{array}$ \\
\hline & Aerobic & $\begin{array}{l}\text { Walking on athletic track } \\
(400 \mathrm{~m})\end{array}$ & $30^{\prime}$ & None & $100 \%$ Critical velocity \\
\hline \multirow{3}{*}{$\begin{array}{l}\text { Intervention } \\
\text { 4: Multimodal } \\
\text { training }\end{array}$} & Dual task & Exercises with accessories & $15^{\prime}$ & 1 ' & 30 '" (three rounds with four bases) \\
\hline & Strength & $\begin{array}{l}11 \text { exercises for strength } \\
\text { with training equipment }\end{array}$ & $27^{\prime}$ & 1 ' & $\begin{array}{l}\text { Weeks } 1-4: 3 \times 15 \text { repetitions } 65-70 \% 1 \mathrm{RM} \\
\text { Weeks } 5 \text { to } 8: 3 \times 12 \text { repetitions } 70-75 \% 1 \mathrm{RM}\end{array}$ \\
\hline & Aerobic & $\begin{array}{l}\text { Walking on athletic track } \\
(400 \mathrm{~m})\end{array}$ & $15^{\prime}$ & None & $100 \%$ Critical velocity \\
\hline
\end{tabular}


repeated to determine and adjust the intensities for the next 4 weeks of training. Participants were instructed to drink water and wear appropriate shoes and clothing during the training. The rating of perceived exertion was determined at the end of each session ${ }^{19}$.

\section{Intervention 2: Functional training}

The functional training was developed using circuit exercises that consisted of 11 sets of exercises (three exercises either with coordination, balance or agility exercises and eight exercises with strength exercises). The coordination and agility exercises were performed using equipment such as ladder drills, cones and hurdle runs. The balance exercises used Bosu ${ }^{\circledR}$ Swiss balls and proprioception platforms. The strength exercises used the same muscular groups as the concurrent training, with free weights and elastic bands (Teraband $\AA$ ). Details and illustrations of these protocols are available in another study from our group ${ }^{20}$. A 30-second interval was allowed between each set, and the participants were required to perform three complete circuits during the training, followed by a 15-minute walk. For the first 4 weeks, a fixed time of 40 seconds was proposed for the performance of each exercise. After that, to maintain progress the exercise time in each set was increased to 50 seconds. The training load was determined using the subjective stress scale Borg's scale ${ }^{19}$, with reference to intensities 12 and 13 (moderate intensity). It is well documented that such intensities (12 and 13 on the Borg scale) correspond to moderate exercise indicators, a fact that supports our choice ${ }^{21}$. The performed exercises had the objective of developing the following: strength capabilities (abdominal, biceps curl, lateral raise, seated row, bending knees, crucifix, triceps handle, squat), agility (displacement among cones), coordination (movement on the agility ladder) and proprioception (one foot on the ground and one on a board). CV was used to determine walking intensity.

\section{Intervention 3: Concurrent training}

The concurrent group developed strength exercises (strength training) followed by aerobic exercise, 27 minutes used for resistance training and 30 minutes for aerobic training (this is a similar load to the aerobic protocol ${ }^{15}$ ). The exercises in the resistance training program worked the same muscle groups as the functional group, but using traditional equipment, and included the following exercises: $45^{\circ}$ leg press, leg extension, knee flexion with ankle weights, bench press, rowing low on cable, direct threaded bar, triceps and abdominal tests. The resistance training program consisted of two progressive phases [phase 1 (1st to 4th weeks, 12-15 repetitions, three sets per exercise, 60 sec between sets); phase 2 ( 5 th to 8 th weeks, 10-12 repetitions, three sets per exercise, $60 \mathrm{sec}$ between sets); the training load was adjusted after four training weeks in order to maintain the prescribed number of repetitions.

The intensity of the resistance training was controlled through repetition maximum (RM). The series were executed until momentary exhaustion, meaning that the participants performed the training with repetitions varying from 12 to $15 \mathrm{RM}$, always being stimulated to execute at least 12 and not more than 15 repetitions ${ }^{22}$. In the case that a participant executed more than 15 repetitions, the load was increased in order to respect the training zone ${ }^{22}$.

\section{Intervention 4: Multimodal training}

The Multimodal group developed a training session composed of proprioceptive training with dual task (15 minutes), followed by strength exercises ( 27 minutes) and walking (15 minutes).

The proprioceptive training consisted of 12 exercises, three for each of the following components: 1 . Coordination; 2 . Agility; 3. Proprioception and Balance; 4. Posture. In coordination exercises, subjects worked motor coordination in upper and lower limbs separated and simultaneously. Hula-hoops and small balls were used to work the upper limbs and for lower limbs the subjects did different exercises in the agility ladder (the exercises in the agility ladder was the same used in functional training, following the preview article published ${ }^{20}$. To exercise as a dual task (two motor tasks simultaneously) we used the same instruments, and in general the subjects either needed to walk while they bounced the ball or used the hula-hoops on their arms. In the last weeks with the exercises progressive, subjects did exercises either walking or running throwing small balls for the subject who accompanied her (exercise in pairs). In agility exercises we emphasized changing direction and velocity and changes in postures (for example, subjects began the agility circuit seated, they should walk or run between cones and hurdle runs, they needed to flex the knees to touch a cone in the floor and finalize the agility circuit seated). In section three, subjects performed proprioception and balance exercises on stable and unstable surfaces (bosu, proprioceptive disc, balance boards, tires) in dynamic positions (performed in gait in tandem on different surfaces) and quiet positions. The quiet position was done by varying the base of support (feet separated, both feet together, semi tandem and tandem position, single leg stance), and was associated or not with trunk movements (for example: women should do rotation of trunk while they needed to be quiet in a bosu). In postural exercises, muscle activity of the abdominals were important to get a better posture and balance; the Swiss ball, bosu and proprioceptive discs were used. Subjects remained 1 minute and 30 seconds on each base: 30 seconds performing each exercise and 1 minute of rest before the next. Therefore, they performed three complete circuit sets, totaling 15 minutes. The training load of functional circuit training was determined as well as functional training using the subjective stress scale Borg's scale $^{19}$, with reference to intensities 12 to 13 (moderate intensity).

\section{Statistical Analysis}

Levene's test was used to analyze data homogeneity. To compare the average relative differences between groups $(\Delta \%)$, one-way ANOVA was used and if a significant difference between groups was observed then a Tukey post-hoc test was conducted. Linear mixed models were used to compare the different training modalities. When a significant difference or interaction was 
observed, a Tukey post-hoc test was performed. For all measured variables, the estimated sphericity was verified according to the Mauchly's W test and the Greenhouse-Geisser correction was used when necessary. The effect size was calculated for all variables (ES). All analyses were performed using BioEstat (version 5.0) statistical software. The significance level was set at $5 \%$. The general physical fitness index was calculated (GPFI) according to the proposal of Zago and Gobbi ${ }^{23}$.

\section{Results}

Table 1 presents the mean values of age, height, fat mass and functional fitness at baseline in the different groups analyzed. It can be seen that there were statistical differences only in strength and cardiorespiratory capacity in the functional training group.

Table 2 presents the changes in the functional fitness values after the 8-week-training protocols. There was a main effect of time for coordination $(F=26.528 ; p<0.001, E S=0.32)$, with a statistically significant interaction $(\mathrm{F}=4.520, p=0.003)$, however there were no differences between groups $(F=0.851$, $p=0.499)$. For flexibility, there were no main effects of time $(F=0.078 ; p=0.781, E S=0.001)$, differences between groups or interaction $(p>0.05)$.

For strength, there was a main effect of time $(F=9.264$; $p=0.004, E S=0.14)$, but there were no differences between groups or interaction $(p>0.05)$. For agility, there was a main effect of time $(F=47.513 ; p<0.001, E S=0.45)$, however, there were no differences between groups or interaction $(p>0.05)$.

Table 1. General characteristics of the groups at baseline.

\begin{tabular}{|c|c|c|c|c|c|c|}
\hline Variables & Control $(n=16)$ & $\operatorname{Aerobic}(n=9)$ & Concurrent $(n=9)$ & Multimodal $(n=14)$ & Functional $(n=14)$ & $p$-value \\
\hline Age (years) & $59.1 \pm 4.9$ & $63.3 \pm 7.1$ & $59.0 \pm 6.6$ & $58.3 \pm 3.6$ & $59.0 \pm 4.9$ & 0.2 \\
\hline Height (cm) & $154.5 \pm 6.1$ & $155.9 \pm 4.2$ & $155.6 \pm 7.4$ & $156.1 \pm 5.4$ & $156.0 \pm 5.6$ & 0.9 \\
\hline Weight (kg) & $68.4 \pm 12.3$ & $65.4 \pm 7.4$ & $68.1 \pm 6.0$ & $68.6 \pm 13.4$ & $66.7 \pm 10.2$ & 0.9 \\
\hline BMI $\left(\mathbf{k g} / \mathrm{cm}^{2}\right)$ & $28.61 \pm 4.78$ & $26.93 \pm 2.97$ & $28.96 \pm 3.28$ & $28.06 \pm 4.78$ & $27.33 \pm 3.41$ & 0.8 \\
\hline Fat mass (kg) & $31.0 \pm 9.3$ & $33.3 \pm 12.5$ & $31.6 \pm 4.0$ & $31.6 \pm 9.6$ & $29.6 \pm 7.6$ & 0.9 \\
\hline Fat mass (\%) & $44.4 \pm 6.2$ & $45.0 \pm 3.1$ & $46.5 \pm 5.0$ & $45.3 \pm 6.2$ & $43.8 \pm 5.5$ & 0.8 \\
\hline Coordination (sec) & $11.0 \pm 2.1$ & $10.4 \pm 1.4$ & $11.4 \pm 1.8$ & $11.0 \pm 1.7$ & $12.8 \pm 2.9$ & 0.1 \\
\hline Flexibility (cm) & $46.0 \pm 10.9$ & $54.4 \pm 12.4$ & $49.1 \pm 7.3$ & $47.6 \pm 13.1$ & $51.2 \pm 11.0$ & 0.4 \\
\hline Strength (rep) & $22.4 \pm 4.3$ & $20.6 \pm 3.0$ & $22.3 \pm 4.1$ & $23.0 \pm 3.4$ & $17.4 \pm 3.9$ acd & 0.002 \\
\hline Agility (sec) & $23.2 \pm 2.5$ & $23.8 \pm 4.3$ & $23.4 \pm 2.8$ & $23.5 \pm 2.6$ & $23.3 \pm 3.6$ & 0.9 \\
\hline $\begin{array}{l}\text { Cardiorespiratory } \\
\text { capacity (sec) }\end{array}$ & $524.2 \pm 55.6$ & $501.7 \pm 47.8$ & $513.0 \pm 35.5$ & $501.4 \pm 33.3$ & $469.8 \pm 37.9^{\mathrm{a}}$ & 0.022 \\
\hline
\end{tabular}

Tukey's post-hoc test with $p$-value $<0.05$ compared to the control group; $\mathrm{b}=$ Tukey's post-hoc test with $p$-value $<0.05$ compared to the concurrent group; $\mathrm{c}=$ Tukey's post-hoc test with $p$-value $<0.05$ compared to the multimodalgroup.

Table 2. Characteristics of the participants before and after the exercise program in different capacities.

\begin{tabular}{|c|c|c|c|c|c|c|c|c|c|c|}
\hline \multirow[t]{2}{*}{ Variables } & \multicolumn{2}{|c|}{ Control $(n=16)$} & \multicolumn{2}{|c|}{ Aerobic $(n=9)$} & \multicolumn{2}{|c|}{ Concurrent $(n=9)$} & \multicolumn{2}{|c|}{ Multimodal $(n=14)$} & \multicolumn{2}{|c|}{ Functional $(n=14)$} \\
\hline & Pre & Post & Pre & Post & Pre & Post & Pre & Post & Pre & Post \\
\hline \multirow{3}{*}{ Coordination (sec) } & 11.0 & 10.1 & 10.4 & 10.5 & 11.4 & 10.3 & 11.0 & 8.9 & 12.8 & 9.4 \\
\hline & \pm & \pm & \pm & \pm & \pm & \pm & \pm & \pm & \pm & \pm \\
\hline & 2.1 & 2.8 & 1.4 & 2.3 & 1.8 & 0.7 & 1.7 & 1.1 & 2.9 & 2.1 \\
\hline \multirow{3}{*}{ Flexibility (cm) } & 46.0 & 48.9 & 54.4 & 52.3 & 49.1 & 51.8 & 47.6 & 50.1 & 51.2 & 48.9 \\
\hline & \pm & \pm & \pm & \pm & \pm & \pm & \pm & \pm & \pm & \pm \\
\hline & 12.3 & 8.0 & 12.4 & 13.4 & 7.3 & 10.4 & 13.1 & 9.5 & 11.0 & 7.0 \\
\hline \multirow{3}{*}{ Strength (rep) } & 22.4 & 22.6 & 20.6 & 20.3 & 22.3 & 23.9 & 23.0 & 24.4 & 17.4 & 22.4 \\
\hline & \pm & \pm & \pm & \pm & \pm & \pm & \pm & \pm & \pm & \pm \\
\hline & 4.3 & 2.9 & 3.0 & 3.1 & 4.1 & 2.8 & 3.4 & 2.6 & 3.9 & 4.8 \\
\hline \multirow{3}{*}{ Agility (sec) } & 23.2 & 22.9 & 23.8 & 20.5 & 23.4 & 21.3 & 23.5 & 20.5 & 23.3 & 19.7 \\
\hline & \pm & \pm & \pm & \pm & \pm & \pm & \pm & \pm & \pm & \pm \\
\hline & 2.5 & 2.8 & 4.3 & 3.6 & 2.8 & 3.4 & 2.6 & 2.1 & 3.6 & 2.7 \\
\hline \multirow{3}{*}{$\begin{array}{l}\text { Cardio. Capacity } \\
\text { (time/sec) }\end{array}$} & 524.2 & 518.4 & 501.7 & 467.9 & 513.0 & 481.9 & 501.4 & 483.5 & 469.8 & 456.1 \\
\hline & \pm & \pm & \pm & \pm & \pm & \pm & \pm & \pm & \pm & \pm \\
\hline & 55.6 & 46.2 & 47.8 & 41.4 & 35.5 & 42.1 & 33.3 & 33.0 & 37.9 & 37.4 \\
\hline
\end{tabular}

Values are expressed as mean \pm standard deviation. Statistical difference over time, ${ }^{*} \mathrm{p}<0.05 ; * * \mathrm{p}<0.001$. a $=$ Tukey’s post-hoc test with $\mathrm{p}$-value $<0.05$ compared to the control group; $b=$ Tukey's post-hoc test with $\mathrm{p}$-value $<0.05$ compared to the multimodal group. 
For cardiorespiratory capacity, there was a main effect of time $(F=32.179 ; p<0.001, E S=0.36)$, with a statistically significant interaction $(F=2.834, p=0.033)$ but there were no differences between groups $(F=0.764, p=0.533)$.

Table 3 presents the relative mean differences between groups $(\Delta \%)$ after 8 weeks of training and the comparisons between the groups.

Statistically significant differences were observed between the functional training group and control group regarding coordination $(p=0.006)$ and there were significant differences in strength when comparing the functional, aerobic, multimodal and control groups $(p=0.005)$. Agility was decreased in the aerobic, multimodal and functional training groups in relation to the control group $(p=0.005)$. There were no statistically significant differences in flexibility $(p=0.417)$ or cardiorespiratory capacity $(p=0.063)$ between the different training programs conducted. Table 4 presents the General Physical Fitness Index (GPFI) with normative values for postmenopausal woman.

The GPFI demonstrates the changes in the different groups after the intervention.

Table 3. Comparison between groups in the relative different capacities $(\Delta \%)$.

\begin{tabular}{|c|c|c|c|c|c|c|}
\hline Variables & Control $(n=16)$ & Aerobic $(n=9)$ & Concurrent $(n=9)$ & Multimodal $(n=14)$ & Functional $(n=14)$ & $p$-value \\
\hline$\Delta$-Coordination & 8 & -1 & 10 & 19 & $27^{\mathrm{a}}$ & 0.006 \\
\hline$\Delta$-Flexibility & 6 & -4 & 5 & 5 & -4 & 0417 \\
\hline$\Delta$-Strength & 1 & -1 & 7 & 6 & $29^{\mathrm{a}, \mathrm{b}, \mathrm{c}}$ & 0.005 \\
\hline$\Delta$-Agility & 1 & $14^{\mathrm{a}}$ & 9 & $13^{\mathrm{a}}$ & $15^{\mathrm{a}}$ & 0.005 \\
\hline$\Delta$-Cardiovascular capacity & 1 & 7 & 6 & 4 & 3 & 0.063 \\
\hline
\end{tabular}

$\mathrm{a}=$ Tukey's post-hoc test with $p$-value $<0.05$ compared to the control group; $\mathrm{b}=$ Tukey's post-hoc test with $p$-value $<0.05$ compared to the aerobic group; $\mathrm{c}=$ Tukey's post-hoc test with $p$-value $<0.05$ compared to the multimodal group

Table 4 General Physical Fitness Index (GFFI) according to the proposal of Zago and Gobbi, 2003 with normative values for postmenopausal woman proposed by Neves, Fortaleza, Rossi, Diniz, Buonani, Ferreira \& Freitas Júnior, 2016

\begin{tabular}{|c|c|c|c|c|c|c|c|c|c|c|}
\hline \multirow[t]{2}{*}{ Variables } & \multicolumn{2}{|c|}{ Control $(n=16)$} & \multicolumn{2}{|c|}{ Aerobic $(\mathrm{n}=9)$} & \multicolumn{2}{|c|}{ Concurrent $(n=9)$} & \multicolumn{2}{|c|}{ Multimodal $(n=14)$} & \multicolumn{2}{|c|}{ Functional $(n=14)$} \\
\hline & Pre & Post & Pre & Post & Pre & Post & Pre & Post & Pre & Post \\
\hline Coordination (sec) & 61 & 85 & 57 & 79 & 61 & 83 & 61 & 86 & 59 & 83 \\
\hline Flexibility (cm) & 50 & 57 & 49 & 54 & 51 & 55 & 53 & 58 & 50 & 55 \\
\hline Strength (rep) & 70 & 79 & 64 & 74 & 66 & 77 & 67 & 78 & 68 & 77 \\
\hline Agility (sec) & 67 & 83 & 59 & 78 & 64 & 82 & 69 & 85 & 63 & 83 \\
\hline Cardiovascular Capacity (sec) & 57 & 70 & 59 & 69 & 59 & 71 & 60 & 73 & 59 & 71 \\
\hline Moment & Total & Class & Total & Class & Total & Class & Total & Class & Total & Class \\
\hline Pre & 305 & Good & 288 & Regular & 301 & Good & 310 & Good & 299 & Regular \\
\hline Pós & 374 & Good & 354 & Good & 368 & Good & 380 & Good & 369 & Good \\
\hline
\end{tabular}

According to Zago and Gobbi (2003), a value of 0 to 100 is assigned for the result of each variable, and these are then summed for the attributes coordination, flexibility, strength, agility and cardiorespiratory capacity, resulting in a single value. Very weak 0-100; Weak 101-200; Regular 201-300; Good 301-400; Very good 401-500

\section{Discussion}

The objective of this study was to compare different modalities of training on coordination, flexibility, strength, agility, and cardiorespiratory capacity in women over 50 years of age and to investigate which training program is more effective for promoting significant improvements in physical fitness. The main finding of this study was that functional training demonstrated the greatest number of significant changes when compared to the other groups. In addition, for the variables coordination, strength, agility and cardiorespiratory capacity, there was a main effect of time, and for the variables coordination and cardiorespiratory capacity there was a statistically significant interaction.

Agility is defined as a rapid whole-body movement with changes in velocity or direction in response to a stimulus ${ }^{24}$, having a close relation with reduction in falls ${ }^{25}$. Aerobic, strength, flexibility and balance training programs are regularly recommended to decrease falls ${ }^{26,27}$. Besides the importance of developing this capacity, the presented data is in line with the results of other studies that investigated the effects of exercise in improving agility ${ }^{10,28}$.

Regarding coordination, exploration of this capacity was included in the protocols of both the multimodal and functional exercise groups, which was not true of the aerobic and concurrent interventions, thus justifying the findings. Physical activity is associated with better coordination as noted in participants of long-term programs ${ }^{26}$, however, the data presented in the present study demonstrates that short-term interventions can also be successful for this capability. It seems that the type of activity influences this variable.

For the variable cardiorespiratory capacity, only the functional training group did not demonstrate a significant response, even though functional training was conducted in circuit form. This group showed significant differences at baseline when compared to the other groups, presenting the highest level of cardiorespiratory capacity, which may have influenced the non-significance of the results after the intervention. 
Regarding relative strength, it is important to note that the specificity of the strength test involved in the AAHPERD battery has a strong influence on strength endurance, a situation exploited by the intervention strategy with functional training exercises that approximated the related movements with the test. The AAHPERD battery has another limitation as the test does not assess the strength of the lower limbs. Furthermore, the low values observed at the initial moment in the functional group may have influenced the significance found among the other groups.

Both strength and cardiorespiratory capacity are shown to decline with aging ${ }^{1,2}$ because a decrease in muscular strength and number and size of type II fibers accompanies a decline in muscle mass related to age ${ }^{29}$. In relation to progressive aging, cardiopulmonary capacity is associated with biochemical changes in the muscle cells, including a reduction in skeletal muscle cells and decrease in the myosin heavy chain I and IIa fibers and muscle oxidative capacity of the damaged muscle ${ }^{30}$.

When comparing the relative differences between exercise protocols (Table 3), the functional training group presented greater improvement values compared to the other groups regarding coordination, strength and agility, these values are significantly better than the control group for the variables coordination $(27 \%$ improvement) and agility (15\% improvement). Functional training also presented greater improvements than the control, aerobic exercise and concurrent training groups for the variable strength (29\% improvement). The multimodal (12\% improvement) and aerobic training groups (14\% improvement) presented significantly higher values than the control group for agility.

Table 4 shows the rating of the overall physical fitness index, where the aerobic and functional groups changed from a regular rate to a good rate, while the control group maintained a regular rate in both evaluations.

The present study compared different training strategies and demonstrated that exercise promoted improvements in physical fitness. Furthermore, functional training proved to be more efficient for improving physical fitness than the other groups.

The use of indirect evaluation is a limitation of this study. Furthermore, the number of participants and the non-randomization between groups is another important limitation.

However, the findings highlighted here - this being one of the first studies to use different training protocols to compare the effects of different stimuli on the functional fitness of older women - demonstrate the potential of exercise to attenuate the decline in functional fitness.

\section{Conclusion}

Functional training was demonstrated to be more effective at promoting significant improvements in physical fitness than the other investigated exercise protocols.

These findings are of great value as the functional decline caused by aging is an important problem for this population, and the main study proposal was to compare four types of exercise interventions.

\section{References}

1. Auyeung TW, Lee SW, Leung J, Kwok T, Woo J. Age-associated decline of muscle mass, grip strength and gait speed: a 4-year longitudinal study of 3018 community-dwelling older Chinese. Geriatr Gerontol Int. 2014; 14 Suppl 1: 76-84.

2. Hicks GE, Shardell M, Alley DE, Miller RR, Bandinelli S, Guralnik J, et al. Absolute strength and loss of strength as predictors of mobility decline in older adults: the InCHIANTI study. J Gerontol A Biol Sci Med Sci. 2012; 67(1): 66-73.

3. Breton E, Beloin F, Fortin C, Martin A, Ouellet ME, Payette H, Levasseur M. Gender-specific associations between functional autonomy and physical capacities in independent older adults: results from the NuAge study. Arch Gerontol Geriatr. 2014; 58(1): 56-62.

4. Osness WH. Functional Fitness Assessment for Adults Over 60 Years (A Field Based Assessment): ERIC. 1990.

5. Caspersen CJ, Powell KE, Christenson GM. Physical activity, exercise, and physical fitness: definitions and distinctions for health-related research. Public health reports. 1985; 100(2): 126.

6. Oppewal A, Hilgenkamp TI, van Wijck R, Schoufour JD, Evenhuis HM. Physical fitness is predictive for a decline in daily functioning in older adults with intellectual disabilities: results of the HA-ID study. Res Dev Disabil. 2014; 35(10): 2299-2315.

7. Cristi C, Collado PS, Marquez S, Garatachea N, Cuevas MJ. Whole-body vibration training increases physical fitness measures without alteration of inflammatory markers in older adults. Eur J Sport Sci. 2014; 14(6): 611-619.

8. Cruz-Ferreira A, Marmeleira J, Formigo A, Gomes D, Fernandes J. Creative Dance Improves Physical Fitness and Life Satisfaction in Older Women. Res Aging. 2015.

9. Gouveia ER, Maia JA, Beunen GP, Blimkie CJ, Fena EM, Freitas DL. Functional fitness and physical activity of Portuguese community-residing older adults. J Aging Phys Act. 2013; 21(1): 1-19.

10. Seguin RA, Heidkamp-Young E, Kuder J, Nelson ME. Improved physical fitness among older female participants in a nationally disseminated, community-based exercise program. Health Educ Behav. 2012; 39(2): 183-190.

11. Zhuang J, Huang L, Wu Y, Zhang Y. The effectiveness of a combined exercise intervention on physical fitness factors related to falls in community-dwelling older adults. Clin Interv Aging. 2014; 9:131-140.

12. Purath J, Keller CS, McPherson S, Ainsworth B. A randomized controlled trial of an office-based physical activity and physical fitness intervention for older adults. Geriatr Nurs. 2013; 34(3): 204-211.

13. Cadore EL, Izquierdo M. How to simultaneously optimize muscle strength, power, functional capacity, and cardiovascular gains in the elderly: an update. Age (Dordr). 2013; 35(6): 2329-2344.

14. Mazo GZ, Benedetti TRB, Gobbi S, Ferreira L, Lopes MA. Valores normativos e aptidão funcional em homens de 60 a 69 anos de idade. Rev Bras Cineantropom Desempenho Hum. 2010; 12(5): 316-323.

15. Rossi FE, Fortaleza AC, Neves LM, Buonani C, Picolo MR, Diniz TA, et al. Combined Training (Aerobic Plus Strength) Potentiates a Reduction in Body Fat but Demonstrates No Difference on the Lipid Profile in Postmenopausal Women When Compared With 
Aerobic Training With a Similar Training Load. J Strength Cond Res. 2016; 30(1): 226-234.

16. Wakayoshi K, Yoshida T, Udo M, Harada T, Moritani T, Mutoh Y, Miyashita M. Does critical swimming velocity represent exercise intensity at maximal lactate steady state? Eur J Appl Physiol Occup Physiol. 1993; 66(1): 90-95.

17. Takahashi S, Wakayoshi K, Hayashi A, Sakaguchi Y, Kitagawa K.. A method for determining critical swimming velocity. Int $\mathbf{J}$ Sports Med. 2009; 30(2): 119-123.

18. Pollock M, Gaesser G, Butcher J, Després J, Dishman R, Franklin B, Garber CE. American College of Sports Medicine Position Stand. The recommended quantity and quality of exercise for developing and maintaining cardiorespiratory and muscular fitness, and flexibility in healthy adults. Med Sci Sports Exerc. 1998; 30(6): 975-991.

19. Borg G, Hassmen P, Lagerstrom M. Perceived exertion related to heart rate and blood lactate during arm and leg exercise. Eur J Appl Physiol Occup Physiol. 1987; 56(6): 679-685.

20. Neves LM, Fortaleza AC, Rossi FE, Diniz TA, Codogno JS, Gobbo LA, et al. Functional training reduces body fat and improves functional fitness and cholesterol levels in postmenopausal women: a randomized clinical trial. 2015. The Journal of sports medicine and physical fitness. J Sports Med Phys Fitness. (in press).

21. Scherr J, Wolfarth B, Christle JW, Pressler A, Wagenpfeil S, Halle M. Associations between Borg's rating of perceived exertion and physiological measures of exercise intensity. Eur J Appl Physiol. 2013; 113(1): 147-155.

22. Silva CMd, Gurjão ALD, Ferreira L, Gobbi LTB, Gobbi S. Efeito do treinamento com pesos, prescrito por zona de repetições máximas, na força muscular e composição corporal em idosas. Rev Bras Cineantropom Desempenho Hum. 2006; 8(4): 39-45.

23. Zago AS, Gobbi S. Valores normativos da aptidão funcional de mulheres de 60 a 70 anos. Revista Brasileira de Ciência e Movimento. 2003; 11(2): 77-86.

24. Sheppard JM, Young WB. Agility literature review: Classifications, training and testing. Journal of sports sciences. 2006; 24(9): 919-932.

25. Liu H, Song L, Zhang T. Changes in brain activation in stroke patients after mental practice and physical exercise: a functional MRI study. Neural Regen Res. 2014; 9(15): 1474-1484.

26. Nelson ME, Rejeski WJ, Blair SN, Duncan PW, Judge JO, King AC. et al. Physical activity and public health in older adults: recommendation from the American College of Sports Medicine and the American Heart Association. Med Sci Sports Exerc. 2007; 39(8): 1435-1445.
27. Panel on Prevention of Falls in Older Persons, A. G. S., \& British Geriatrics, S. Summary of the Updated American Geriatrics Society/British Geriatrics Society clinical practice guideline for prevention of falls in older persons. J Am Geriatr Soc. 2011; 59(1): 148-157.

28. Emerenziani GP, Meucci M, Gallotta MC, Buzzachera CF, Guidetti L, Baldari C. Whole body vibration: unsupervised training or combined with a supervised multi-purpose exercise for fitness? J Sports Sci. 2014; 32(11): 1033-1041.

29. Granacher U, Gollhofer A, Hortobagyi T, Kressig RW, Muehlbauer T. The importance of trunk muscle strength for balance, functional performance, and fall prevention in seniors: a systematic review. Sports Med. 2013; 43(7): 627-641.

30. Vigorito C, Giallauria F. Effects of exercise on cardiovascular performance in the elderly. Front Physiol. 2014; 5: 51.

\section{Acknowledgments}

We would like to thank all the staff of the Motor Activity Lab and Prescription Center-CELAPAM.

\section{Corresponding author}

Lucas Melo Neves

CELAPAM (Motor Activity Research Lab and Prescription Center), Department of Physical Education, Universidade Estadual Paulista, UNESP, Educational Center, 305, Roberto Simonsen st, Presidente Prudente, SP, Brazil.

Email: lucasmeloneves@uol.com.br

Manuscript received on December 09, 2015

Manuscript accepted on May 16, 2016

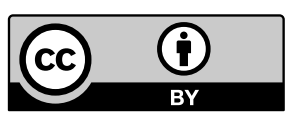

Motriz. The Journal of Physical Education. UNESP. Rio Claro, SP, Brazil - eISSN: 1980-6574 - under a license Creative Commons - Version 3.0 